\title{
La concepción del tiempo en la cultura mapuche*
}

\author{
Maria Ester Grebe
}

\section{INTRODUCCIÓN}

En las culturas de Occidente predomina una concepción regular y continua del tiempo. Este se representa en un modelo cronológico compartido, homogéneo y uniforme (Bohannan, 1967:328). Medimos el tiempo en unidades constituidas artificialmente, aceptadas consensualmente, validadas y legitimadas por la tradición cultural. Su flujo puede ser medido mediante instrumentos específicos creados por el hombre, tales como relojes y calendarios. Esta concepción continua del tiempo depende de un sistema de conexiones cualitativas que conducen a la división de duraciones y a las operaciones cuantitativas (Hallp1Ke, 1979:347). Una vez internalizada esta concepción temporal, que implica una percepción culturalmente específica del paso abstracto del tiempo medido en unidades siempre iguales y constantes, ella invade nuestras vidas, organizando y coordinando nuestras actividades. En el transcurso de nuestra existencia urbana se nos presentan dos opciones: amoldar y coordinar nuestras actividades a su implacable secuencia o luchar contra ésta con el fin de maximizar el rendimiento y lograr las metas propuestas. Esta concepción del tiempo es una construcción cultural occidental que opera, generalmente, en un nivel subliminal demarcando nuestra existencia y devenir.

No obstante, "la regularidad del tiempo no es parte intrínseca de la naturaleza, sino una noción creada por el hombre que hemos proyectado en nuestro ambiente para nuestros propósitos particula-

*El presente trabajo fue presentado en el Segundo Congreso Argentino de Antropología Social, Buenos Aires, Universidad de Buenos Aires, 1986 , e incluido en el Simposio $\mathrm{N}^{\circ} 8$ Etnología y Lingüística presidido por el Dr. Edgardo Cordeu. Su marco teórico se ha publicado separadamente en la revista Lenguas Modernas (Universidad de Chile, Santiago, véase GREBE, $1987:$ 163-171. 
res" (Leach, 1979:227). De ello se infiere que la concepción regular y continua del tiempo en perpetuo movimiento no es universal. Es, más bien, una concepción particular que caracteriza apropiadamente a los sectores urbanos afines al desarrollo del mundo de Occidente. En suma, las representaciones o modelos de tiempo o de procesos temporales son relativos y varían de acuerdo a la cultura y subcultura, aun cuando es posible detectar la presencia de factores comunes y diferenciados que caracterizan tanto a la concepción de tiempo occidental, como al así llamado "tiempo primitivo". En uno y otro caso, el reconocimiento del tiempo es un proceso cognitivo complejo de percepción, conceptualización y simbolización, que incluye tanto la clasificación y demarcación como la representación de los fenómenos temporales.

Una caracterización de las concepciones de tiempo de las sociedades occidentales y primitivas debe considerar que tal dicotomía posee límites difusos y puede estar afectada por criterios etnocéntricos del hombre de Occidente. Por tanto, la caracterización que proponemos a continuación debe ser considerada provisoria y tentativa, quedando sujeta a revisiones críticas ulteriores.

Frente a la concepción occidental del tiempo regular, continuo, homogéneo y uniforme, que utiliza mediciones basadas en operaciones cuantitativas e instrumentales, la concepción primitiva del tiempo se centra en un dominio perceptivo en el cual predomina un reconocimiento intuitivo de la duración y sucesión de fenómenos mediante operaciones predominantemente cualitativas. Ambas concepciones podrían ser el resultado de diferencias de desarrollo tecnológico en los procedimientos de medición del tiempo disponibles en las sociedades humanas respectivas (Goody, 1977:45). La concepción primitiva del tiempo aparece intimamente ligada a una concepción espacial y kinésica asociada a factores tales como la distancia recorrida, el trabajo realizado y la velocidad (HALLPIKE, loc. cit.). Se incorpora así la concepción primitiva del tiempo en un contexto procesual (BarNes, 1971:541), en el que destacan las secuencias particulares concretas de los eventos del medio ambiente natural y social; $y$, particularmente, las interacciones recurrentes de los hombres en y con dichos contextos.

Mientras la concepción occidental del tiempo se da preferentemente en sociedades modernas que se identifican con los patrones culturales y subculturales prevalecientes en el mundo urbano, la concepción primitiva del tiempo es propia de sociedades tradicionales campesinas e indígenas, que se identifican con los patrones culturales y subculturales del mundo rural. La migración rural-urbana implicaría una coexistencia de ambos modelos temporales, expresada ya sea en uso simultáneo de éstos, o bien en un proceso de cambio transicional y adaptativo.

En el presente trabajo se estudiará la representación colectiva del tiempo de los mapuches de Chile, que comparte diversas características con la concepción primitiva del tiempo recién descrita como 
propia de las sociedades tradicionales indígenas y campesinas. Esta concepción del tiempo será examinada de acuerdo a las distinciones percibidas por los propios ind ígenas y a las categorías empleadas por ellos. Asimismo, se considerarán las ordenaciones del fenómeno temporal, reflejadas en los conceptos de dominio, conjuntos de contraste y sus significados simbólicos, en la medida en que éstos se incorporan en los etnomodelos elaborados por los mapuches. Estos etnomodelos describen, analizan y explican las divisiones temporales de dos ciclos:

a) del día y noche; b) de las estaciones del año (cf. Ohnuk i-TieRNEY, 1973:287-291).

El trabajo en terreno se desarrolló intensamente en el curso de los años 1985-1988 en reducciones mapuches próximas al río Cautín. La secuencia metodológica empleada es flexible y abierta, a diferencia del método cognitivo ortodoxo. En líneas generales, incluyó las siguientes etapas:

1. Exploración y rapport.

2. Apertura de canales de comunicación.

3. Producción de documentos primarios por el indígena.

4. Rescate de conceptos de dominio, etnocategorías, conjuntos de contraste y de sus significados simbólicos decodificados por el indígena.

5. Incentivación de la descripción y análisis producidos por el indígena.

6. Incentivación de la formulación de modelos explicativos generados por el indígena.

7. Elaboración del propio modelo explicativo del antropólogo. obviamente en un plano epistemológico diferente.

\section{El tiempo MAPUChe}

Dos lexemas básicos opuestos se asocian respectivamente a la concepción del tiempo pasado y presente: kuifi (remoto, antiguo, viejo) y $w e$ (nuevo, reciente, joven). El pasado se vincula al tiempo remoto de los mitos de origen, de dioses y demonios, como asimismo al tiempo antiguo de antepasados, héroes y chamanes fallecidos. El presente se vincula al flujo temporal percibido, el cual se desdobla en dos conceptos expresados en mapuche y español: 1. antü (sol), "el tiempo mapuche de día y de noche"; en otras palabras, el ciclo del día y sus divisiones, y 2. tripantü (año), "los tiempos del año mapuche", que equivale al ciclo de las estaciones.

El futuro existe, pero en función del peuma (sueño), que posee generalmente un carácter premonitorio; del pewutun, rito de diagnóstico por adivinación, y del trance extático, en el cual el chamán comunica vaticinios o profecías.

Los mapuches no practican el cómputo de días y carecen de la noción de semana. Durante el día, el tiempo se capta en las posiciones 
del sol y de los rayos solares (Hilger, 1974:344), y durante la noche por las posiciones de ciertos astros, entre los cuales se destacan, en calidad de indicadores de tiempo, los siguientes: wünelfe (lucero del alba), yepun (lucero de la noche), welu-witrau (cruz del sur) y ngau (constelación astral de estrellas pequeñas).

En el presente trabajo nos ocuparemos preferentemente del concepto del tiempo presente, centrando nuestro estudio en dos etnomodelos producidos en calidad de documentos primarios consensuales por mapuches residentes en reducciones tradicionales próximas a Quepe y Metrenco. Dichos etnomodelos, que representan los ciclos día-noche y estaciones del año, fueron producidos en forma espontánea y segura en el lugar de residencia de los informantes. Los documentos primarios recogidos fueron examinados, analizados y comparados, tomando en cuenta los testimonios entregados por informantes calificados e idóneos, todos ellos buenos conocedores de los conocimientos, creencias y prácticas tradicionales de su propia cultura.

El nivel de rapport y los lazos de amistad de la antropóloga que suscribe este trabajo, producto de veinte años de experiencia en terreno, contribuyeron a crear un medio ambiente humano propicio para las interacciones y sus expectativas, todo lo cual benefició el desarrollo exitoso de este trabajo. El conocimiento previo acumulado por esta investigadora sobre praxis ritual, mitología y simbología mapuche fueron muy valiosos para crear un ambiente de reciprocidad en el trabajo de recolección, como asimismo para permitir y estimular el establecimiento de relaciones entre los diversos fenómenos en estudio y gravitar en su red de significados.

El etnomodelo de la fig. 1 representa a antü (sol), el ciclo dianoche, traducido como "tiempo mapuche de día y de noche". Es una representación clara y elocuente de la concepción espacial y kinésica del tiempo mapuche. Al centro del diagrama se ubica la tierra mapuche con su forma cuadrada tradicional, meli witran mapu, la tierra de las cuatro esquinas o lugares (Grebe, 1972:50; 1973:26). Esta plataforma terrestre se orienta hacia el este (puel mapu), punto cardinal optimo, desglosando la tetrapartición de los cuatro puntos cardinales. Esta plataforma integra un conjunto de siete plataformas iguales, divididas de acuerdo a dicha tetrapartición, que componen el cosmos mapuche (Grebe, 1972:50). Por tanto, los ejes espaciales simétricos de los cuatro puntos cardinales orientan y dividen la representación del tiempo mapuche.

Alreded or del esquema espacial central del diagrama aparece un círculo con doce puntos que demarcan divisiones temporales. Cada una de éstas corresponde a una categoría temporal designada con el correspondiente lexema nativo. En torno a este círculo aparecen flechas circulares que representan el circuito completo del ciclo diario. Es un tiempo circular reversible de eterno retorno (ELIADE, 1967: 71, 77), que descansa en dos ejes simétricos y representa, según los testimonios recibidos, al movimiento del sol alrededor de la tierra. 


\section{Figura 1}

\section{EL CICLO DIA-NOCHE EN LA CULTURA MAPUCHE} (Etnomodelo)

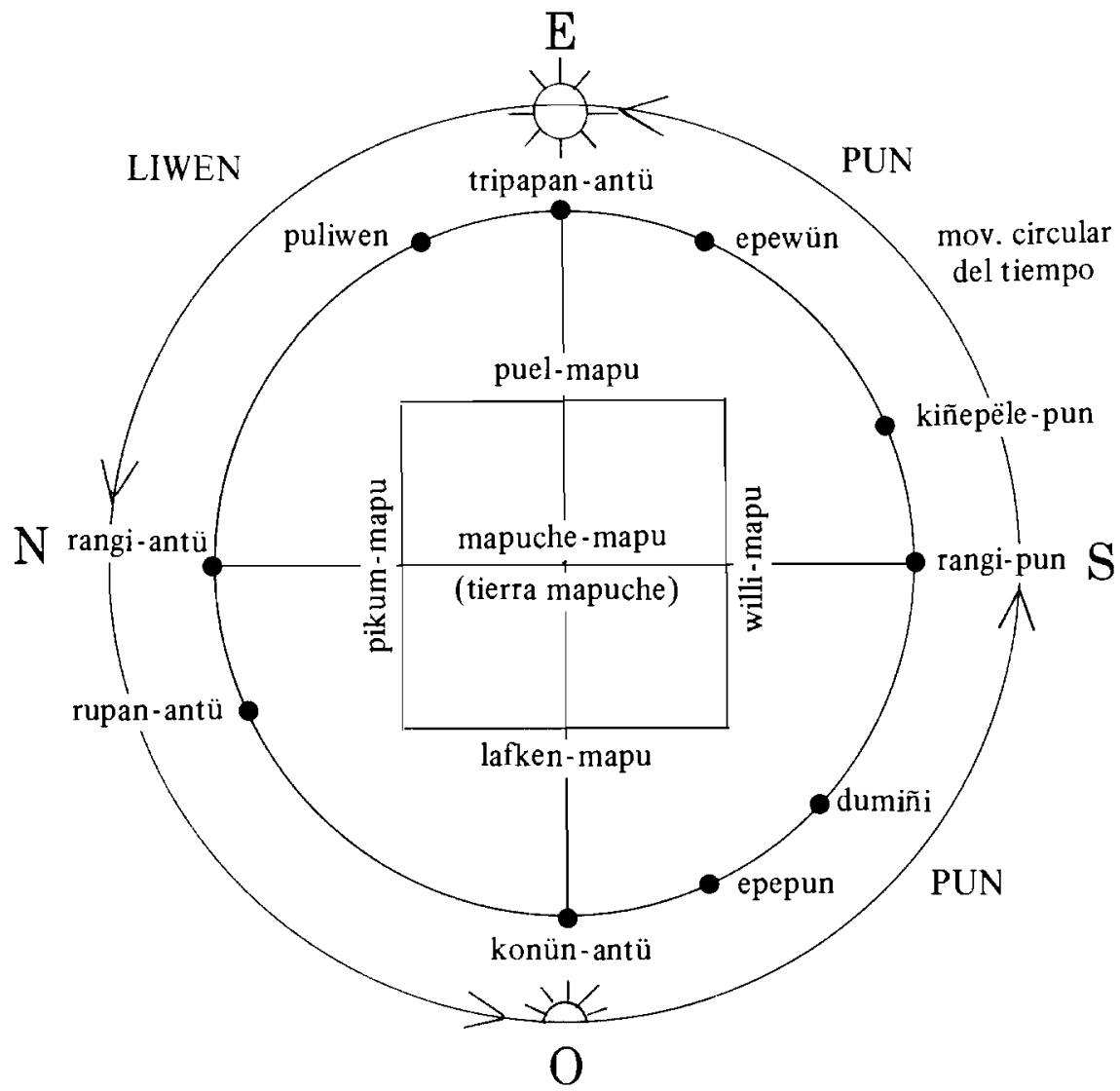

Las doce categor ías temporales mapuches son:

1. epewün: alba o amanecer.

2. tripapan-antü: salida del sol.

3. puliwen: después de la salida del sol.

4. liwen: mañana.

5. rangi-antü: mediodía o cenit.

6. rupan-antü o rupan-rangi-antü: después de mediodía.

7. konün-antü: puesta del sol.

8. epepun: crepúsculo o atardecer.

9. dumiñi: oscuridad.

10. pun: noche.

11. rangi-pun: medianoche.

12. kiñepële-pun: después de medianoche. 
Cabe señalar que estos lexemas varían de un área geográfica a otra, como lo atestigua una comparación de datos etnográficos proporcionados por Moesbach (1936:82-84), Ccoper (1946:754), Hilger (1957:84-86, 310) y Benigar (1978:88-93). Los contenidos que se representan a continuación corresponden a relaciones, asociaciones y significados de estos lexemas, proporcionados por los propios informantes y complementados por referencias bibliográficas:

1. Epewün marca el advenimiento gradual de la claridad y la luz. Es un periodo de máximas connotaciones positivas asociado a los dioses y al momento trascendente de la creación. El nacimiento del nuevo día reactualiza cotidianamente y en pequeña escala la creación del universo mapuche. En este contexto, la luz del alba se asocia a las fuerzas positivas generadoras de nueva vida. Se afirma que "el mapuche se siente feliz, con alegría, cuando sale la luz: hay esperanza y deseo de un día mejor". Ayong es el blanco transparente del alba, color simbólico que representa esencialmente al bien y al poder benéfico de las deidades del wenu-mapu, las cuatro plataformas del mundo sobrenatural benéfico (GREBE, 1972: 50, 59-60). El indicador de la llegada del alba es la aparición de wünyelfe-kushe, la anciana mensajera del lucero del alba, quien baja a la tierra mapuche transmitiendo el mensaje de los dioses del wenu-mapu a la machi (chamán) durante su sueño. Entonces la machi despierta súbitamente y ejecuta el rito solitario del pillantun frente a su rewe, en el cual se comunica con los espíritus de antiguas machis fallecidas, espíritus auxiliares y dioses.

2. Tripapan-antü corresponde a la salida del sol, representado por su primer rayo. Marca el fin del descanso nocturno, la iniciación de la jornada matinal y de las actividades cotidianas. En el sistema de creencias mapuches el sol posee connotaciones ambivalentes. Por un lado, se le considera una bola de fuego destructiva, asociada a la sequía, fuego, fiebre y color rojo; y, por otro, se le relaciona con la acción benéfica de fertilización y germinación de la tierra y del reino vegetal. Esto último explica las invocaciones dirigidas al sol como antü-fücha y antü-kushe (dios anciano y diosa anciana del sol), los cuales mantienen aún su vigencia en algunas reducciones mapuches tradicionales (FARON, 1964:53). Asimismo, la luz solar se relaciona con los antüpaiñamko (águilas venidas del sol), que representan a los espiritus de los antepasados (FARON, 1964:4). De esto se desprende que el primer rayo del sol evoca estos contextos positivos de fertilidad, germinación y divinidades benefactoras. Pero, por sobre todo, evoca el quiebre o derrota de las tinieblas de la noche asociadas al mal. Debido a estas connotaciones, tripapan-antü, es el momento propicio para iniciar actividades rituales.

3. Puliwen es la segunda parte de la mañana (liwen), reconociéndose cuando el sol está alto. Es tiempo propicio para el trabajo agrícola y/o pastoril. 
4. Liwen es el período matinal más extenso, que abarca desde el alba hasta el mediodía, incluyendo la salida del sol. Se le considera la mejor parte del día, por cuanto se produce un gran despliegue de energía y vitalidad manifiesta en el desarrollo de actividades tanto productivas como creativas en general. Se suele afirmar que "hay más energía para trabajar y para iniciar algo: trabajo, viaje, ceremonia". Es el período cotidiano de mayor productividad que el mapuche intenta aprovechar cabalmente. Por esta razón, es común que los mapuches más emprendedores se levanten temprano o de madrugada, hecho al que se asigna gran valor. Este patrón cultural mapuche suele ser compartido, respetado y valorado tanto por las familias, grupos de descendencia y amigos como por la comunidad mapuche tradicional como un todo.

5. Rangi-antü no equivale exactamente a nuestro concepto occidental de mediodía (12 horas), sino a un período de duración mayor aproximada de una hora a partir de las 12 del día. Se reconoce, porque el sol está en su punto más alto (cenit) y "pega más fuerte". Suele ser medido por las sombras que proyectan ciertos objetos. En la práctica, este período transcurre más o menos desde las 12 a 13 horas de cada día, lapso destinado al almuerzo familiar y al descanso que prosigue. Se traduce al español como "la hora de doce", lo cual se ha prestado a equívocos en el curso del trabajo en terreno. En efecto, cuando un informante cita al antropólogo para "la hora de doce", éste suele llegar sin estar invitado cuando la familia almuerza. El antropólogo se percata, entonces, de que su cita era para después del almuerzo, lo cual lo ayudará a no incurrir nuevamente en el mismo error. Este incidente se generó debido a una comprensión defectuosa de las categorías de tiempo mapuche por parte del antropólogo.

6. Rupan-antü o rupan rangi-antü se extiende desde pasado el mediodía hasta antes del atardecer. Durante este período se reinician las actividades laborales comenzadas en la mañana. No obstante, la energía y productividad tienden a decrecer. "Es un tiempo más flojo. Se va acabando la fuerza. Se va cansando el día y la persona". Por esta razón, "el que empieza [o se levanta] después de doce no es alentado". Este comentario indica que rupan-antü es una fase secundaria destinada a actividades productivas que se subordina a liwen por su menor intensidad y/o disminución del ritmo de trabajo.

7. Konün-antü es la puesta de sol que cierra el eje vertical del ciclo cotidiano, cuyo punto de partida fue el nacimiento del sol (tripapan-antü). Si el nacimiento del sol representa simbólicamente la vida y la energía, la puesta del sol representa lo opuesto: el sol y la energía vital declinan y desaparecen para renacer al día siguiente dando nuevo impulso al ciclo cotidiano.

8. Epepun es el período correspondiente al atardecer o crepúsculo. Marca el término de las actividades laborales cotidianas que coinciden con la disminución gradual de la luz. En el transcurso de este período, "siente el mapuche tranquilidad, reposo y descanso". El ciclo diario de 
la luz ha llegado a su término. Y con ello se produce una división simétrica del día en dos mitades caracterizadas respectivamente por la luz (dominio del bien) y la oscuridad (dominio del mal). En la fase final del epepun, se produce un cierre simbólico de la mejor parte del día. Es el momento propicio para dar término a diversas actividades rituales, particularmente las terapéuticas, puesto que se cree que la disminución de la luz favorece el regreso de los malos espíritus a su ámbito originario: la oscuridad. “¡Tardei, tardei!”, es la exclamación que expresa la preocupación por el crepúsculo que avanza. Si hay visitas en el hogar, el deber de un buen anfitrión es ofrecerles hospedaje para evitar los peligros de un viaje nocturno. Después de aclarar el nuevo dia, las visitas pueden partir sin riesgos.

9. Dumiñi marca el advenimiento de la oscuridad. "Cae la noche, no hay más luz. Es tiempo de dormir, de soñar, de pensar en lo que se va a hacer al día siguiente". Por estas razones, los miembros de la familia mapuche buscan resguardo de los peligros de la noche, refugiándose en su vivienda. Dentro de ésta se sienten protegidos de un medio ambiente externo oscuro, incierto, inseguro y no controlado por el hombre. Tienden a permanecer dentro de la vivienda, como una medida preventiva contra la oscuridad asociada al mal. Evitan viajar de noche por caminos solitarios. Sólo en una situación de emergencia sería posible viajar acompañado, pero no solo.

10. Pun o puni es el periodo nocturno más extenso que abarca desde el crepúsculo tard io hasta antes del amanecer. Se cree que "es, en general, malo: desde dumiñi hasta kiñepële-pun es tiempo malo de wekufe y kalku". Durante este período el hombre mapuche pierde el control del medio ambiente externo. Es un mundo hostil preñado de peligros que acechan. Es el reino del mal, cuyo principal agente humano es el kalku (brujo), quien se cree que opera clandestinamente en la noche, cobijándose en el renü, su cueva subterránea, donde manipula a los wekufes (espíritus malignos) y programa sus malas artes $\mathrm{y}$ acciones nefastas.

11. Rangi-pun difiere de nuestro concepto occidental de medianoche (cero horas). Consiste en un período de duración aproximada de una hora, a partir de las doce de la noche, que se extiende hasta el advenimiento de kiñepële-pun (después de medianoche). Se le considera un tiempo de máximo peligro: "Es tan peligroso que se siente miedo. Es la hora en que salen los wekufes y kalkus". Se cree que estos agentes del mal -que, por lo general, permanecen ocultos-se hacen visibles y deambulan durante el rangi-pun a través del entorno próximo a las viviendas mapuches, particularmente en senderos solitarios, bosques, matorrales, pantanos, ríos o lagunas.

12. Kiñepële-pun se extiende desde después de medianoche hasta el amanecer (epewün). La peligrosidad previa sigue vigente, pero ésta va decreciendo en la medida en que se aproxima el amanecer. Este es un período transicional, que marca el fin del ciclo nocturno y sus connotaciones negativas. En la fase final del kiñepële-pun se produce 
un cierre simbólico de la mitad oscura del ciclo diario asociada al mal, ratificándose la división simétrica del día en dos mitades opuestas: el día, asociado al bien y a los dioses; la noche, asociada al mal, a los espíritus malignos y brujos. Esta dicotomía, que corresponde a indicaciones de tiempo generadas por fenómenos naturales y a los significados atribuidos a sus conjuntos básicos de contrastes, suele reaparecer con frecuencia en las concepciones temporales de diversas culturas tradicionales. En general, los conceptos de tiempo determinados por el ambiente forman parte de la cosmología, reflejando las relaciones del hombre con el entorno. Evans Pritchard (1977:111-155) los denomina concepciones ecológicas del tiempo.

\section{B. Ciclo anual de las estaciones (véase cuadro 1)}

En la cultura mapuche el año nuevo no comienza el primero de enero, fecha establecida por el calendario oficial de Occidente. En efecto, winoitripantü, el año nuevo mapuche, se inicia "cuando los días comienzan a ser más largos que las noches" (HILGER, 1966:120). Su punto de partida se identifica con el solsticio de invierno, que se celebra a partir de la noche del 23 de junio (víspera), culminando el día 24 de junio que corresponde al día de San Juan. Este día marca la iniciación del calendario anual mapuche y de su correspondiente ciclo de las estaciones.

Sin embargo, la división del calendario anual en estaciones y sus denominaciones respectivas (lexemas) varían flexiblemente de acuerdo a las peculiaridades de los cambios de la naturaleza, factores climáticos y actividades agrícolas de cada región (HILGER, 1966:120; Bragg, 1984: 180). En general, las estaciones del año no poseen puntos fijos precisos de iniciación y término, puesto que los fenómenos naturales, tales como clima, temperatura y lluvias, tienden a variar de un año a otro.

Así, en Pangupulli el calendario anual se divide en siete estaciones (HILGER, loc. cit.):

1. we tripantü: se extiende desde el año nuevo hasta el tiempo para sembrar (loc. cit.); es "el tiempo en que vuelve el sol del norte" (Augusta, 1966: 245) y equivale al fin del invierno. siembra.

2. wün'n tripantü: primavera, tiempo de crecimiento de la

3. antü tripantü o antüngen: verano, tiempo de sol y de calor.

4. mon mapu: tiempo de cosecha, de tierra fértil, de madurez de los frutos y de abundancia de comida.

5. chomüngen: otoño, tiempo posterior a la cosecha.

6. konme pukem: preinvierno.

7. pukem: invierno.

En la zona pehuenche el ciclo anual se divide en dos estaciones principales: walüng (verano) y pukem (invierno). Mientras walüng 
TIEMPOS DEL AÑO MAPUCHE (ETNOMODELO)

(El ciclo de las estaciones)

\begin{tabular}{|c|c|c|c|c|}
\hline $\begin{array}{l}\text { Estaciones del año } \\
\text { mapuche }\end{array}$ & $\begin{array}{l}\text { We tripantï } \\
\text { (privamera) }\end{array}$ & $\begin{array}{l}\text { Walüng tripantü } \\
\text { (verano) }\end{array}$ & $\begin{array}{l}\text { Nganüwen } \\
\text { (otoño) }\end{array}$ & $\begin{array}{l}\text { Pukem } \\
\text { (invierno) }\end{array}$ \\
\hline Meses correspondientes & $\begin{array}{l}\text { Octubre, noviembre, } \\
\text { diciembre }\end{array}$ & Enero, febrero, marzo & Abril, mayo, junio & Julio, agosto, septiembre \\
\hline $\begin{array}{l}\text { Actividades productivas } \\
\text { y fenómenos climáticos }\end{array}$ & $\begin{array}{l}\text { Tiempo de floreci- } \\
\text { miento }\end{array}$ & $\begin{array}{l}\text { Tiempo de cosecha y } \\
\text { calor }\end{array}$ & Tiempo de siembra & Tiempo de lluvia y frio \\
\hline Connotaciones & $\begin{array}{l}\text { küme kiyen } \\
\text { (meses buenos) }\end{array}$ & $\begin{array}{l}\text { arre kiyen } \\
\text { (meses de calor) }\end{array}$ & $\begin{array}{l}\text { küme kiyen } \\
\text { (meses buenos) }\end{array}$ & $\begin{array}{l}\text { weda kiyen } \\
\text { (meses malos) }\end{array}$ \\
\hline $\begin{array}{l}\text { Actividades religiosas } \\
\text { y sociales }\end{array}$ & $\begin{array}{l}\text { ngillatun } \\
\text { ngeikurrewen } \\
\text { (Tiempo de ritos de } \\
\text { fertilidad, iniciáticos } \\
\text { y postiniciáticos) }\end{array}$ & $\begin{array}{l}\text { përamuwün } \\
\text { (Tiempo para recoger } \\
\text { la cosecha) }\end{array}$ & $\begin{array}{l}\text { ngillatun } \\
\text { ngeikurrewen } \\
\text { (Tiempo de ritos de } \\
\text { fertilidad, iniciáticos } \\
\text { y postiniciáticos) }\end{array}$ & $\begin{array}{l}\text { derfiwün } \\
\text { (Tiempo para cuidarse y } \\
\text { protegerse) }\end{array}$ \\
\hline $\begin{array}{l}\text { Colores de la } \\
\text { naturaleza }\end{array}$ & karrë (verde) & chod (amarillo) & kadü (café) & kürrë (negro) \\
\hline $\begin{array}{l}\text { Vientos y sus } \\
\text { connotaciones }\end{array}$ & $\begin{array}{l}\text { waiwen kürrëf } \\
\text { (Viento sur, trae } \\
\text { buen tiempo) }\end{array}$ & $\begin{array}{l}\text { waiwen kürrëf } \\
\text { (Viento sur, trae } \\
\text { buen tiempo) }\end{array}$ & $\begin{array}{l}\text { konpa kürrëf } \\
\text { (Viento norte, trae } \\
\text { mal tiempo) } \\
\text { puelche } \\
\text { (Viento este, trae } \\
\text { buen tiempo) }\end{array}$ & $\begin{array}{l}\text { konpa kürrëf } \\
\text { (Viento norte, trae mal } \\
\text { tiempo) } \\
\text { lafken kürrëf } \\
\text { (Viento del mar, trae } \\
\text { mal tiempo) }\end{array}$ \\
\hline
\end{tabular}


coincide con la residencia en tierras altas y el calor, pukem se relaciona con la permanencia en tierras bajas y el frío (BRAGG, 1984:179). A su vez, walüng y pukem contienen respectivamente cinco y tres períodos, formando un total de ocho períodos cuyas denominaciones son coincidentes con aquellas de Panguipulli (Hilger, 1966:120):

1. walüng: verano.

a) wün'n tripantü: noviembre, diciembre.

b) antü tripantü: enero.

c) antügnen: febrero.

d) mon mapu: marzo.

e) chomüngen: abril, mayo (otoño).

2. pukem: invierno.

a) konme pukem: mayo, junio 24 .

b) pukem: junio 24 , julio, agosto.

c) we tripantü: septiembre, octubre (primavera).

Ambas estaciones poseen indicadores ligados a cambios del medio ambiente físico. Así, "pukem termina el día en que las golondrinas vuelven a Icalma, entre el 10 y el 15 de septiembre". Y walüng se inicia cuando las nevazones disminuyen, a partir de noviembre (BraGG, 1984: 180). Compárese los datos etnográficos recién citados de Hilger (1966:120) y Bragg (1984:180) con aquellos de Hilger (1957:87-88) y Benigar (1978:94-98).

En una reducción mapuche del valle central, vecina al río Quepe, aparece una versión simplificada del ciclo de las estaciones, recogida por la presente investigadora en el verano de 1986. Esta versión se reproduce esquemáticamente en el siguiente cuadro 1 , generado en un etnomodelo producido por los propios actores nativos (véase el etnomodelo en el cuadro adjunto).

Un primer análisis de este esquema indica que la división en cuatro estaciones es coincidente a aquella de la cultura occidental. En un primer examen parecería constituir una versión simplificada y aculturada de esta última. Pero un análisis más detallado demuestra que las relaciones establecidas desde el punto de vista nativo en el etnomodelo con las actividades sociales productivas y religiosas como también con fenómenos naturales -clima, vientos, colores- pertenecen a una concepción del mundo netamente mapuche. Así, la pareja de oposición bien-mal constituye un centro de gravitación y principio dominante del ciclo estacional, dando sentido a una trama compleja de relaciones significativas (GREBE, 1972, 1973).

En este caso, küme kiyen -los meses buenos de primavera y otoño- constituyen las estaciones propicias tanto para ciertas actividades productivas agrícolas (siembra y cosecha) como también para desarrollar la mayor parte de los rituales religiosos. A la inversa, weda kiyen -los meses malos de invierno- conforman una estación apropiada para el resguardo contra las inclemencias del exceso de lluvia y 
frío, siendo el cuidado de la salud una preocupación central. Es tiempo propicio para desarrollar actividades artesanales al interior de la vivienda. Por su parte, arre kiyen - los meses calurosos del veranose dedican prioritariamente a las tareas agrícolas de recolección de diversos tipos de cosecha: manzanas, avena y cebada en enero; trigo, arvejas y habas en febrero; papas, maíz y porotos en marzo.

\section{CONCLUSIONES Y DISCUSIÓN}

De los resultados precedentes es posible inferir las siguientes conclusiones:

1. Los mapuches carecen de un lexema equivalente a "tiempo" en su lengua nativa. Ellos no experimentan la sensación de luchar contra el tiempo ni tampoco coordinar rígidamente sus actividades en función de éste. Su ritmo vital de acción es pausado, sin apremios, sin presiones ni tensiones. “'Apurruwai!”, o ¡apúrate!, es un lexema que expresa una categoría sincrética champurria, préstamo cultural derivado del español.

2. La percepción temporal cuantitativa centrada en unidades o divisiones de tiempo abstractas y exactas es ajena a los mapuches rurales pertenecientes a reducciones tradicionales. Ellos manejan una concepción cualitativa del tiempo ligada a fenómenos naturales y sociales; a cambios ecológicos que reflejan sus relaciones con el medio ambiente físico y cambios estructurales que reflejan sus relaciones mutuas en las actividades sociales (Evans.PRITCHARD, 1977:111-155).

3. Los fenómenos naturales proporcionan puntos de referencia explícitos al pasar de un estado a otro y al integrarse a un ritmo vital que tiene sentido de acuerdo a preceptos compartidos colectivamente por los mapuches. Dichos puntos de referencia suelen ser la dirección y variación de los vientos; los cambios climatológicos (frío o calor, lluvia o sequía); los cambios en la vegetación y sus colores; los movimientos y posición de los cuerpos celestes; la migración de algunas especies de pájaros, etc.

4. Los fenómenos sociales proporcionan otros puntos de referencia relevantes, al enmarcar el fenómeno temporal en un dominio semántico cargado de significados y símbolos. Dichos fenómenos sociales aparecen intimamente vinculados a los cambios naturales del medio ambiente físico. En efecto, tanto las actividades sociales productivas como los ritos mapuches ocurren en el contexto de las divisiones del ciclo diario y de los cambios estacionales que integran el calendario anual tradicional, compartiendo una red de significados que les dan coherencia y sentido cultural específico.

5. El hombre mapuche ha vivido, y vive, en contacto permanente e íntimo con la naturaleza. Su interacción cotidiana con los diversos fenómenos naturales es intensa y cargada de significados. La tierra, el agua, el bosque, la montaña, la piedra, el viento y otros elementos 
aparecen animados. Para el mapuche, poseen vida propia, energía y espiritualidad. Y esa animación espiritual transforma a la naturaleza a través de los dos ciclos temporales en estudio: del día a la noche, de la primavera al invierno. Observador atento de dichos cambios, el hombre mapuche ve reflejado el paso del tiempo en el espejo de la naturaleza.

6. Las actividades religiosas del pueblo mapuche están enmarcadas también en los dos ciclos descritos y sus connotaciones. Esto es particularmente evidente en el caso del tiempo sagrado de los ritos. En efecto, sólo los meses "buenos" de primavera y otoño son considerados propicios para los ritos comunitarios. El tiempo óptimo para la iniciación de actividaes rituales es el epewün (alba) que coincide con la aparición de la luz asociada a los dioses, a la creación y al bien. Para poner término a dichas actividades, se considera propicio el epepun (crepúsculo), que coincide con la desaparición de la luz y el fin del dominio de los dioses y del bien.

7. La gran mayoría de las actividades sociales y productivas, tales como la siembra y la cosecha, la caza y la recolección, el pastoreo, los viajes, las visitas a parientes y amigos y ciertas actividades domésticas y artesanales se rigen por la elección de estaciones y horas del día propicias, cuyas connotaciones positivas se asocian al bien. La elección del tiempo justo y adecuado asegura el éxito de las actividades a desarrollar.

8. Los mapuches poseen una concepción espacial y kinésica del tiempo, en la cual tiempo, espacio y movimiento se interpenetran y condicionan recíprocamente. Los indicadores astrales de tiempo-sol, luceros del alba y de la noche, cruz del sur, etc.- señalan tanto el tiempo diurno y nocturno mediante su aparición y posiciones como también el espacio y direccionalidad, proporcionando así orientación al viajero. El tiempo gira de derecha a izquierda en círculos de eterno retorno alrededor del espacio terrestre dividido por los ejes simétricos de los cuatro puntos cardinales. Su movimiento perfecto va contra los punteros del reloj. Así, el tiempo de la salida del sol coincide con el eje espacial del este, y el tiempo de la puesta del sol con el eje espacial del oeste. Ambos comparten respectivamente las connotaciones relacionadas con el bien y el mal, la luz y la oscuridad, la creación y la destrucción, los dioses y los espíritus malignos.

9. El orden explícito de los etnomodelos mapuches en estudio obedece a una necesidad de dar orden y equilibrio al mundo externo en consonancia con los principios fundamentales de la cosmovisión tradicional. El hombre mapuche infiere su concepción del tiempo a partir de su percepción cotidiana del medio ambiente circundante y de los modelos cosmovisionales compartidos, que lo representan. Ambos están filtrados culturalmente e impregnados de una carga semántica consensual.

10. En suma, la concepción del tiempo mapuche es relativa a su base cultural, de la cual se desprende y forma parte. Mientras en 
Occidente prevalece la concepción cuantitativa del tiempo, los mapuches - como muchas otras sociedades tradicionales de América y de los demás continentes- modelan sus concepciones cualitativas del tiempo a partir de su percepción, concepción, categorización y simbolización del medio ambiente natural y social decantado en su visión de mundo.

Estas conclusiones permiten reflexionar, examinar y discutir la concepción de tiempo de la cultura mapuche en el contexto del proceso actual de aculturación en marcha. Surgen algunas interrogantes: ¿Qué grado de continuidad y resistencia al cambio posee la concepción tradicional del tiempo entre los mapuches actuales? ¿Qué influencia ejerce la concepción occidental del tiempo en los mapuches urbanos y rurales, tanto adultos como niños escolares? ¿Cómo afecta la coexistencia de ambas concepciones del tiempo en el proceso de inserción del mapuche en la sociedad mayor?

A pesar de la fuerte continuidad de su tradición cultural, la sociedad mapuche ha debido adaptarse gradualmente a los modelos culturales de la sociedad chilena. La interacción recurrente y convivencia continuada con los chilenos (winkas) tiende a intensificarse en los lugares de estudio y trabajo: escuelas rurales o urbanas, mercados, fundos, ind ustria y comercio, residencias urbanas de chilenos, etc. En todos estos casos, el mapuche necesita internalizar la concepción de tiempo de nuestra cultura para desarrollar sus tareas con eficiencia. Esta necesidad se agudiza en el caso de los migrantes mapuches rural-urbanos que residen en forma permanente o semipermanente en las ciudades.

La compra del primer reloj pulsera parece ser un indicador importante. Es el primer paso dado con el fin de adaptarse e internalizar la concepción del tiempo que prevalece en la sociedad mayor. Sin embargo, estos mapuches conservan su propia concepción tradicional del tiempo al retornar a la reducción de origen. En estos casos, suelen manejarse dos concepciones del tiempo como vías alternativas que se eligen según el medio ambiente y contexto sociocultural.

En nuestros días, el contacto más temprano del niño mapuche rural con nuestra concepción del tiempo se da en la escuela básica rural o de los pueblos vecinos a su reducción. Las profesoras de una escuela básica vecina a Temuco informaron a la presente investigadora (1987) que los escolares mapuches están trabajando y ahorrando dinero con el fin de adquirir dos preciados bienes: una bicicleta y un reloj pulsera. Mientras la bicicleta se utiliza para movilizarse entre el hogar y la escuela, el reloj es el instrumento apropiado y necesario para ajustarse al tiempo medido de la otra cultura.

Los niños mapuches aprenden pronto a ver la hora correctamente, sin equivocaciones. Si bien es cierto que los escolares pequeños de seis a diez años tienen problemas para percibir, medir y organizar cuantitativamente el tiempo, a partir de los diez años van superando sus 
dificultades. Y, al llegar al liceo, han logrado solucionar todos su problemas de ajuste a nuestra percepción del tiempo.

Es difícil prever el grado de adaptación a nuestro tiempo occidental que puede lograr la comunidad mapuche en el futuro. Los procesos de urbanización y aculturación en marcha parecerían predecir una transición sostenida hacia nuestros modelos temporales cuantitativos. Sin embargo, varias características del modelo temporal nativo parecen obstaculizar o dificultar los procesos de transferencia y adaptación. Si bien los mapuches urbanos parecerían ser favorecidos por la adquisición eficiente del modelo de tiempo occidental, los mapuches rurales parecen no necesitarlo aún. En este último caso, prevalece la tendencia conservadora a mantener el tiempo primitivo con sus rasgos cualitativos caracterizadores centrados en su íntima relación con la naturaleza, la cosmología y el medio ambiente social y cultural creado por el hombre. No obstante, este tiempo primitivo no proporciona las condiciones necesarias de apertura para internalizar la concepción occidental del tiempo homogéneo, uniforme y universal.

Estas conclusiones y discusión de alternativas se proponen en calidad de ideas que pueden generar hipótesis de trabajo para investigaciones futuras que traten la concepción de tiempo mapuche en el contexto del proceso de aculturación en marcha.

\section{REFERENCIAS}

Augusta, Félix José de. Diccionario Araucano. Padre Las Casas, San Francisco, 1966.

Barnes, J. A. "Time Flies Like an Arrow". Man (n.s.) [Londres], 6, 1971, pp. $537-552$.

Benigar, Juan. "El Concepto del Tiempo entre los Araucanos". En La Patagonia Piensa, Neuquén, Siringa, 1978, pp. 81-108.

Bohannan, Paul. "Concepts of Time Among the Tiv of Nigeria". En Myth and Cosmos: Readings in Mythology and Symbolism, J. Middleton (ed.), Austin, University of Texas Press, 1967, pp. 315-329.

Bragg, Katherine. "Los Conceptos Lingü1sticos de la División de Espacio, Tiempo y Actividades en una Comunidad Pehuenche". Actas Jornadas de Lengua y Literatura Mapuche [Temuco], Universidad de La Frontera, 1984, pp. 177-188.

Cooper, John M. "The Araucanians". En Handbook of South American Indians, Bur. Amer. Ethnol. Bull, 143, vol. II, Washington, 1946, pp. 687-760.

Eliade, Mircea. Lo Sagrado y lo Profano. Madrid, Ed. Guadarrama, 1967.

Evans-Pritchard, E. E. "El Tiempo y el Espacio". En Los Nuer, Barcelona, Anagrama, 1977, pp. 111-155.

Faron, Louis. Hawks of the Sun. Pittsburgh, University of Pittsburgh Press, 1964.

Goody, Jack. The Domestication of the Savage Mind. Cambridge, Cambridge University Press, 1977.

Grebe, M. Ester. "Cosmovisión Mapuche". Cuadernos de la Realidad Nacional 14 [Santiago], 1972, pp. 46-73. 
Grebe, M. Ester. "El Kultrún Mapuche: Un Microcosmos Simbólico". Revista Musical Chilena [Santiago], 123-124, 1973, pp. 3-42.

Grebe, M. Ester. "Reflexiones Antropológicas Sobre Temporalidad". Lenguas Modernas [Santiago], 14, 1987,pp. 163-171.

Hallpike, G. H. The Foundation of Primitive Thought. Oxford, Clarendon Press, 1979.

Hilger, Inez. Araucanian Child and its Cultural Background. Washington D.C., Smithsonian Institution, 1957.

Hilger, Inez. Huenum Namku. Norman, University of Oklahoma Press, 1966.

Hilger, Inez y Mondloch, Margaret. "Surnames and Time and Distance Measurements Among the Chilean Araucanians". En Native South Americans, Patricia J. Lyon (ed.), Boston, Little/Brown, 1974, pp. 343-345.

Leach, Edmund, "Two Essays Concerning the Symbolic Representation of Time". En Reader in Comparative Religion: An Anthropoligical Approach, W. A. Lessa y E. Z. Vogt (eds.), New York, Harper \& Row, 1979, pp. 221-233.

Moesbach, Ernesto Whilhelm de. Vida y Costumbres de los Indigenas Araucanos en la Segunda Mitad del Siglo XIX, Santiago, Ed. Padre Las Casas, 1936.

Ohnuki-Tierney, Emiko. "Sakhalin Ainu Time Reckoning". Man (n.s.), [Londres] $8,2,1973$, pp. 285-299.

Pocock, David. "The Anthropology of Time-Reckoning". En Myth and Cosmos. Readings in Mythology and Symbolism, J. Middleton (ed.), Austin, University of Texas, 1967, pp. 303-314 